\title{
The protection effect and mechanism of hyperbaric oxygen therapy in rat brain with traumatic injury ${ }^{1}$
}

\author{
Pengcheng Xing', Ke Ma", Lijuan Li"', Donglian Wang'v , Guoyong Hu'v, Wei Long ${ }^{\vee}$
}

'MD, Department of Emergency and Intensive Care Unit, Shanghai Sixth People's Hospital East, China. Acquisition, analysis and interpretation of data; manuscript preparation.

"MD, Department of Emergency and Intensive Care Unit, Shanghai Sixth People's Hospital East, China. Conception and design of the study, manuscript preparation, final approval.

I'MD, Physician, Department of Geriatrics, Shanghai Sixth People's Hospital East, China. Acquisition of data, technical procedures.

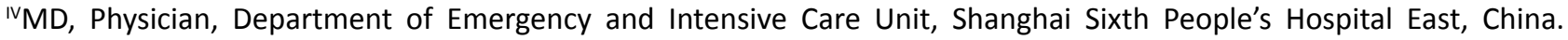
Technical procedures.

'MD, Physician, Department of Geriatrics, Shanghai Sixth People's Hospital East, China. Technical procedures.

\begin{abstract}
Purpose: To investigate the effect of hyperbaric oxygen therapy (HBOT) on traumatic brain injury (TBI) outcome.

Methods: The modified Marmarou's weight drop device was used to generate non-lethal moderate TBI rat model, and further developed in vitro astrocytes culturing system. Then, we analyzed the expression changes of interested genes and protein by quantitative PCR and western blot.

Results: Multiple HBO treatments significantly reduced the expression of apoptosis promoting genes, such as c-fos, c-jun, Bax and weakened the activation of Caspase- 3 in model rats. On the contrary, HBOT alleviated the decrease of anti-apoptosis gene $\mathrm{Bcl}-2$ and promoted the expression of neurotrophic factors (NTFs), such as NGF, BDNF, GDNF and NT-3 in vivo. As a consequent, the neuropathogenesis was remarkably relied with HBOT. Astrocytes from TBI brain or those cultured with $21 \% \mathrm{O}_{2}$ density expressed higher NTFs than that of corresponding controls, from sham brain and cultured with $7 \% \mathrm{O}_{2}$, respectively. The NTFs expression was the highest in astrocytes form TBI brain and cultured with $21 \% \mathrm{O}_{2}$, suggesting a synergistic effect existed between TBI and the following $\mathrm{HBO}$ treatment in astrocytes.

Conclusion: Our findings provided evidence for the clinical usage of HBO treating brain damages.

Key words: Hyperbaric Oxygenation. Nerve Growth Factors. Brain Injuries. Rats.
\end{abstract}




\section{- Introduction}

Traumatic brain injury (TBI) occur when sudden acceleration or deceleration happens within the cranium caused by all kinds of external forces, among which traffic accident is one of the most common and still rising cause in modern society ${ }^{1}$. It is estimated that about 2 million people suffer TBI in the United States annually, accounting for $\sim 30 \%$ injuryrelated deaths $s^{2,3}$. Because of its heterogeneous causes, the signs and symptoms of TBI vary dramatically, depending on the part of brain being affected and severity of the injury. Mild $\mathrm{TBI}$ may cause headache, vomiting, nausea, dizziness, difficulty balancing and so on, while severe TBI may cause convulsions, inability to awaken, slurred speech, aphasia and behavior change in addition ${ }^{1,4}$. According to the pathogenesis feature, TBI damage could be focal or diffused or the combination of both ${ }^{5}$. A focal traumatic injury could be easily detected by conventional imaging method such as CT or MRI, but diffused traumatic injury (DTI) could only be detected by post-mortem microscopy examination or more advanced diffusion tensor imaging MRI techniques ${ }^{6-8}$. According to Granacher J $r^{6}$, DTI includes the following 4 brain damage types: 1) Diffuse axonal injury, which affects the white matter; 2 ) Ischemia, caused by reduced blood supply and a main reason of secondary damage; 3) Vascular injury, and 4) Edema, increasing the intracranial pressure which could be lethal if not treated properly. Normally, these pathological changes do not occur separately; rather, two or more changes occur simultaneously or work as reciprocal causations. Take brain edema for example, which is the most common and rapidly happened symptoms after $\mathrm{TBI}$, combined with vascular injury, it will promote the deterioration of ischemia and further neurodegenerative pathology. Due to the widely spreaded damage and complicated pathogenesis pathways involved, DTI is most challenging for both treatment and prognosis. Though extensive efforts have been devoted to develop therapy methods for TBI/DTI, little was achieved in improving clinical prognosis $s^{9,10}$. Besides the brain damaged caused during acute period, the neuropathologies may sustain and progress into chronic traumatic encephalopathy (CTE), which could be a life-long health threat ${ }^{11}$. TBI associated disabilities affect approximately 5 million people in US with a health care cost over $s \$ 60$ billion $^{12-14}$.

In the last two decades, hyperbaric oxygen therapy (HBOT) has been introduced to treat multiple injuries and disorders, including traumatic ischemia, damage, cerebral palsy and $\mathrm{TBI}$ among others ${ }^{15,16}$. During HBOT, the patient is administered with $100 \%$ oxygen at a pressure greater than atmospheric pressure at sea level within a sealed chamber. The effect of high pressure and increased solubility and diffusion characters of gas $\left(\mathrm{O}_{2}\right)$ are expected to improved oxygenation, vasoconstriction, modulation of inflammation and immune function, and/or promote angiogenesis ${ }^{15}$. In normal tissue, hyperoxia will induce vasculature constriction, but tissue oxygenation remains unchanged because of increased dissolved oxygen ${ }^{17}$. As consequence of vasoconstriction, tissue edema and exposure to reactive oxygen species (ROS), which is induced by hyperbaric oxygen, could be reduced ${ }^{18,19}$. In peripheral tissues such as cartilage and skin, HBOT has been shown to modulate angiogenesis during wound healing ${ }^{20,21}$, whether this is also the case in brain TBI/DTI remain largely unknown. Jiang et al reported that the effect of a single HBO treatment would last for about 12 hours, and therefore suggested multiple HBO treatment could be administered to prolong the response period $^{22}$. Niklas et al. $^{23}$ confirmed that in severe brain TBI model rabbits, multiple HBOT dramatically reduced edema and necrosis areas and consequentially mortality rate.

$$
\text { A meta-analysis including } 12
$$

randomized trials demonstrated that HBOT 
was no better than control treatment for mild TBI; however, HBOT did benefit moderateto-severe TBI for acute treatment. But still, the clinical trial of HBOT was rather limited as the underlining mechanisms remain unclear. In hypoxia-ischemia rat model, a single HBO treatment significantly reduced caspase-3 activity and sequentially reduced apoptotic cell number in both brain cortex and hippocampus ${ }^{24}$. Several lines evidence indicated that TBI induced immediate c-fos and c-jun expression, which were not limited to damage site but rather diffused and the expression were associated with severity of damage ${ }^{25-28}$. Some apoptosis genes, such as Bax was also upregulated following the elevation of c-fos/c-jun, indicating that they may involve in neural death events. There is no direct evidence demonstrating whether HBO treatment improve TBI/DTI prognosis by modulating c-fos/c-jun expression level so far.

In this study, with modified Marmarou rat DTI model, we confirmed that multiple HBOT reduced damage-induced c-fos/c-jun expression and further reduced cell apoptosis. These effects may be partial attributed to the elevated expression of neural trophic factors (NTFs) such as NGF, BDNF, NT3 and GDNF. In vitro cell culturing model demonstrated that astocytes isolated from DTI model rat contributed to the expression elevation of NTFs in response to high $\mathrm{O}_{2}$ concentration, while astocytes isolated from control rat brain were less effective. Our results for the first time connected the neural protective effect of $\mathrm{HBO}$ to NTFs and specified that astocytes as the source of such NTFs.

\section{Methods}

All experimental procedures were performed according to the Guidelines for Animal Care and Use of Shanghai Sixth People's Hospital East.
Adult male SPF Sprague-Dawley rats, 8-week age, weighted $300 \pm 30 \mathrm{~g}$, were purchased from Shanghai Slac laboratory animal CO. Ltd. The rats housed under a 12:12 light-dark cycle with ad libitum feeding.

\section{Modified Marmarou weight drop model}

To induce close head brain diffused traumatic injury, Marmarou weight drop device was adopted with modification ${ }^{29-31}$. First, a metal helmet that match rat skull curve was casted and used to cover the rat head during weight drop, which evenly distribute the vertical force to the whole brain. Second, the weight and falling height of the impactor were careful adjusted to achieve close head moderate DTI. The parameters were strictly fixed to avoided biases among animals. Third, the impactor was connected to a conduction rope, which was manually strained immediately after the impactor contacted the metal helmet to avoid second strike.

Animals were initially anesthetized with $2 \%$ sodium pentobarbital at $45 \mathrm{mg} / \mathrm{kg}$ body weight intraperitoneal. The skull was exposed by midline incision of the dorsal surface and covered with the sterilized metal helmet. Injury was then induced by weight derived falling of the impactor. Sham group rats were only anesthetized and surgically exposed the Skull but without impact injury.

\section{HBO treatment}

After injury, the animals were immediately administered with $\mathrm{HBO}$ treatment of $100 \%$ oxygen at a pressure of 3 atmospheres absolute (ATA) for $1 \mathrm{~h}$, and then returned to normal housing condition. Multiple HBOT was conducted in 12 hour interval for the following 3 days and a total of six treatments were administered. The control group rats were put in the HBO chamber for $1 \mathrm{~h}$ but administered with only normal atmosphere air. 


\section{Assessment of brain edema}

The wet-weight dry-weight method was used to assess brain edema. In brief, at desired time points after injury the whole brains, including bilateral cerebral, diencephalon, mesencephalon, cerebellum and brainstem of 3 animals in each group, were dissected out. The fresh tissues were immediately weighed to get wet weight, then placed at $100^{\circ} \mathrm{C}$ for 24 hours and dry weight were determined. The percentage of water was calculated with the following formulation: $\%$ of $\mathrm{H} 2 \mathrm{O}=$ (wet weight - dry weight)/wet weight $\times 100 \%$.

\section{HE staining}

Paraffin embedding and HE staining were performed as described previously. In short, the animals were perfused with PBS under deep anesthesia, followed by $4 \%$ PAF in PBS for pre-fixation. The brains were removed and placed in the same fixative solution at $4^{\circ} \mathrm{C}$ overnight. Paraffin- embedded brains were sectioned at 5um with a Leica semiautomatic microtome, transferred onto plastic slides and processed for HE staining. Stained slides were mounted with neutral balsam and cover slips, and images were developed with Olympus BX51 microscope equipped with Cellsens software.

\section{Quantitative PCR (qPCR)}

The animals were sacrificed at desired time points and the cerebrums were isolated, separated into left and right hemispheres along midlines, flash frozen in liquid nitrogen $\left(\mathrm{L}_{\mathrm{N} 2}\right)$ and kept at $-80^{\circ} \mathrm{C}$ until use. The left cerebral hemispheres were grind in a mortar pre-cooled with $L_{\mathrm{N} 2 .}$ The samples were then lysed with $1 \mathrm{ml}$ Trizol and total RNA were extracted. 1 ug of each total RNA sample was reverse-transcribed to cDNA using the QuantiTect Reverse Transcription Kit (Qiagen). Real-time PCR was performed with SYBR Green PCR Master
Mix (Applied Biosystems) according to the manufacturer's instruction. All measurements were performed in triplicate and Gapdh mRNA was used to normalize the relative expression levels of target genes.

\section{Western blot}

The right cerebral hemispheres were grind in a mortar pre-cooled with $\mathrm{L}_{\mathrm{N} 2}$ and lysed in RIPA buffer $(25 \mathrm{mM}$ Tris- $\mathrm{HCl} \mathrm{pH} 7.5$, $150 \mathrm{mM} \mathrm{NaCl}, 0.1 \%$ SDS, $0.5 \%$ sodium deoxycholate, $1 \%$ Triton X-100) supplemented with protease cocktail. Equal amounts of protein were separated on $10 \%$ SDS-PAGE gels and transferred onto nitrocellulose membranes. After blocking with $5 \%$ skimmed milk in TBS buffer (50 mM Tris- $\mathrm{HCl}, 150 \mathrm{mM}$ $\mathrm{NaCl})$, membranes were incubated with primary antibodies () diluted in blocking buffer at $4^{\circ} \mathrm{C}$ overnight. The membranes were washed with TBST buffer (TBS $+0.1 \%$ Tween-20) $3 \times 5 \mathrm{~min}$ at room temperature and incubated in corresponding HRP-conjugated secondary antibodies (1:5000; Cell Signaling Technology). The blots were developed using Pierce ECL Western Blotting Substrate Plus and band density was measured with ImageJ software.

\section{Primary astrocyte culturing}

The brains of sham rat and Marmarou weight drop model rat were aseptically dissected, and the meninges were removed. Primary astrocytes were isolated and cultured as described with minor modification ${ }^{32}$. In brief, the cerebrum was chopped to $1 \mathrm{~mm}^{3}$ pieces and digested with $0.05 \%$ trypsin and $0.003 \%$ DNase at $37^{\circ} \mathrm{C}$ for $15 \mathrm{~min}$. The tissue was triturated with fire polished Pasteur pipette, collected, digested with $40 \mathrm{U}$ papain/ $\mathrm{ml}, 0.02 \%$ cysteine and $0.003 \%$ DNase at $37^{\circ} \mathrm{C}$ for $15 \mathrm{~min}$. Then triturated again and filtered through 40 um cell strainer. The single cells 
were collected and re-suspended in DMEM/ F12 supplemented with $10 \%$ FBS and seeded in poly-L-lysine pre-coated dishes at density of $5 \times 10^{5}$ cells $/ \mathrm{cm}^{2}$. To mimic low and high oxygen condition, the cells were kept at $7 \% \mathrm{O}_{2}, 5 \% \mathrm{CO}_{2}$, $88 \% \mathrm{~N}_{2}$ or $21 \% \mathrm{O}_{2}, 5 \% \mathrm{CO}_{2}, 74 \% \mathrm{~N}_{2}$ incubators respectively. After three days incubation, the cells were harvested and mRNA expression was measured with qPCR.

\section{Statistical analyses}

Multiple group were compared using ANOVA (one-way or two-way). Unpaired $t$ tests were used for two-group comparisons. The tests were two-tailed and considered significant when $p<0.05$. All data are presented as mean $\pm S E M$.

\section{- Results}

\section{Modified Marmarou weight drop model}

causes moderate but not lethal TBI

Marmarou's weight drop device was widely used for introducing diffuse axonal injury model of TBI on both rat and mouse since first developed in $1990 \mathrm{~s}^{30}$. We made minor modifications as mentioned above to increase the reproducibility and comparability among animals. With these modifications and carefully adjusted parameters, we got TBI model rats of moderate diffused damages.

We first assessed the brain edema by measuring the percentage of water content, which was about $78 \%$ in sham treated brains and almost no fluctuation observed over time. However, weight drop impact dramatically increased brain water content which was observed as early as 3 hours and peaked at 24 hour after injury, suggesting a severe brain edema and increasing of intracranial pressure (Figure 1A). The incensement of water content was relieved at 3 and 7 days post injury, but still significantly higher than control group, indicating the absorption of edema while pathogenic risk sustained if not treated properly. HE staining and histological examination demonstrated reduced neuronal density in the cerebral cortex at all checked time points (Figure 1B; 3h, 6h, 1d, 3d, 7d after injury) compared with sham control. Shrunken neurons with surrounding vacuolation were observed and the number increased with time, indicating the progress of irreversible neural death. These results were consistent with previous reports ${ }^{29,30,33}$.

A
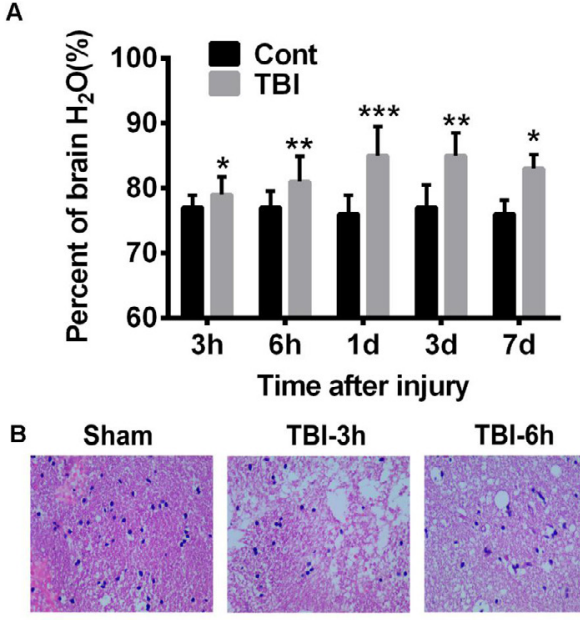

TBI-3h

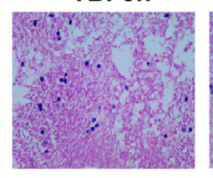

TBI-6h

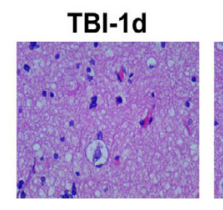

TBI-3d

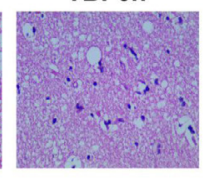

TBI-7d

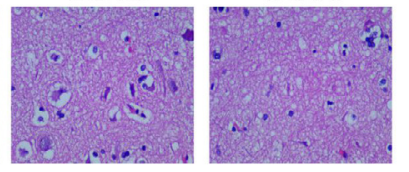

Figure 1 - Modified Marmarou weight drop model successively causes brain edema and neuropathogenesis. A. Brain edema was evaluated by the percentage of water in total brain tissue. In sham treated brains, the water content is about $78 \pm 0.2 \%$. Weight drop impact significantly increases water content which peaks at 24 hours after injury $(82.4 \pm 0.4 \%)$ and decreases but still higher that sham control at 3 and 7 days after injury. ${ }^{*} p<0.05,{ }^{* *} p<0.01$. B. HE staining demonstrates the neuropathogenesis after weight drop impact. Neuron number is decreased, shrunken neurons and peri-neural vacuolation are observed, which aggravate with time. 
TBI elevates apoptosis proteins expression and increases c-fos/c-jun mRNA

It was reported that $\mathrm{TBI}$ induced Caspase-3 activity, which is $32 \mathrm{kD}$ zymogen could be activated by both extrinsic and intrinsic apoptosis pathways ${ }^{34}$. We confirmed that the cleaved $17 \mathrm{kD}$ activity subunit was dramatically increased 24 hours after TBI and sustained to day 7, indicating active apoptosis events (Figure 2A-B). Further, we detected the expression level of proteins $\mathrm{Bcl}$ 2 and Bax, two mutually antagonistic factors of mitochondrial pathway of apoptosis. We found dramatically decreased expression of $\mathrm{Bcl}-2$, which is an anti-apoptosis protein, most apparent at 24 hours after TBI. On the contrary, the expression of Bax, a pro-apoptosis protein which induce mitochondrial outer membrane permeabilization, was markedly increased, as shown in Figure 2A. However, there was no activation of Caspase-3 or Bcl-2/Bax disbalance among the sham treated rats (Figure $2 \mathrm{~A})$. These results suggested that TBI induced neural apoptosis via the intrinsic mitochondrial pathway.

C-fos and c-jun are two immediate-early genes encode proteins that form heterodimer complex AP-1 (Activator Protein-1). Several lines of evidence demonstrated that c-fos/cjun played important roles in apoptosis and the expression of their mRNA could serve as an early indicator of apoptotic events. As expected, compared with sham control, the expression of c-fos/c-jun mRNA were rapidly increased after $\mathrm{TBI}$, reached maximum at 24 hours and drop back to base level at day $3 / 7$ (Figure 2B).

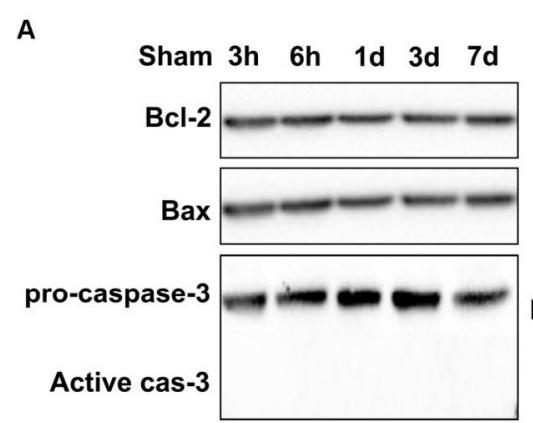

B

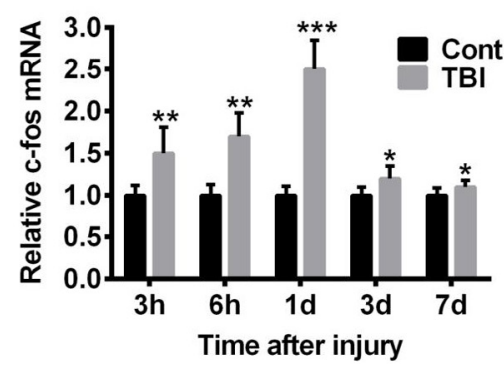

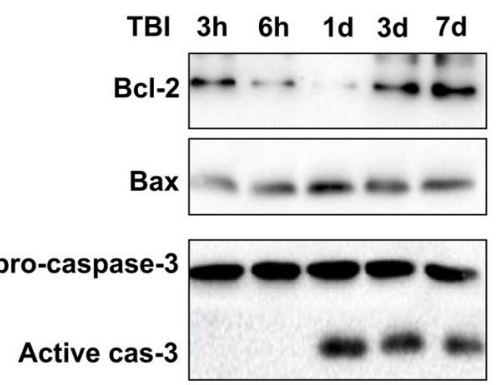

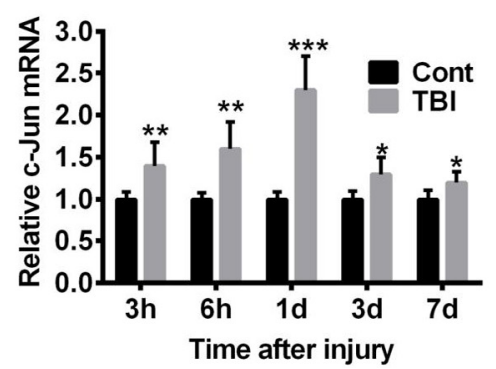

Figure 2 - TBI induces the activation of mitochondria apoptosis pathway. A. Western blot shows the changes of key apoptosis associated proteins. Compared with sham control, TBI reduces the expression of Bcl-2, but promotes the expression of Bax and the activation of Caspase-3. Theses effect is most apparent at time point 24 hours after injury. B. qPCR demonstrates the elevated mRNA expression of c-fos and c-jun, which is most significant at 24 hours after injury. ${ }^{*} p<0.05,{ }^{* *} p<0.01,{ }^{* * *} p<0.005$. 
Multiple HBOT attenuates brain edema and neural pathogenesis induced by TBI

To determine whether hyperbaric oxygen will benefit TBI induced brain damage, the model rats were administrated with multiple HBOT immediately after injury and at 12-hour interval in the following 3 days. Brain samples were collected at time points of $3 \mathrm{~h}$, $6 \mathrm{~h}, 1 \mathrm{~d}, 3 \mathrm{~d}$ and $7 \mathrm{~d}$ post injury, which received 1 (3h, 6h), 2 (1d), 6 (3d, 7d) times of HBOT. As shown in Figure $3 \mathrm{~A}$, HBOT significantly reduced brain water content, which was most apparent at $1 \mathrm{~d}$ after injury, as compared to notreatment controls. HE staining of $1 \mathrm{~d}$ samples demonstrated that HBOT remarkably alleviated the pathological progress, as less shrunken neuron and perineuronal vacuolation was observed (Figure 3B). Consistent with previous report, these results confirmed that HBOT could effectively prevent deterioration of DTI pathogenesis.

A

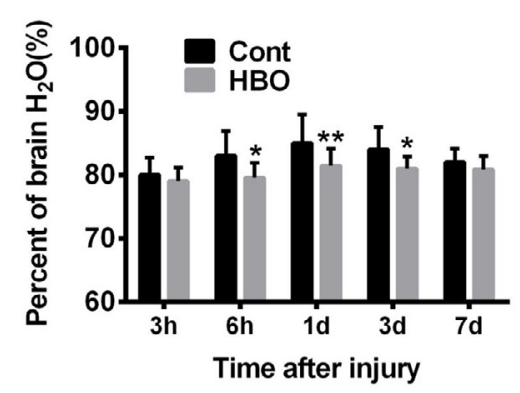

B

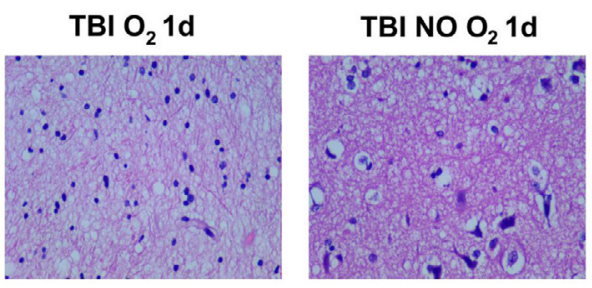

Figure 3 - HBO treatment reduces brain edema and attenuates neuropathogenesis. A. Compared control treatment, HBOT significantly reduced brain edema, as measure by brain water content, at all check time points. ${ }^{*} p<0.05, * * p<0.01$. B. HBOT attenuates neural death as fewer shrunken neurons and peri-neural vacuolation are observed compared with control.
HBOT prevents neural death by inhibiting apoptosis pathway and activating neurotropic factors expression

We further detected the apoptosis protein expression with HBO treated brain samples. The protein expression tendency is similar to TBI brain without HBOT (Figure 2), but several subtle changes were noticed: first, a stronger $\mathrm{Bcl}-2$ band was detected in HBOT group at time point $1 \mathrm{~d}$, which was barely visible in the corresponding TBI sample; second, the increase of Bax expression was less apparent in HBOT group; third, the cleaved Caspase- 3 band was more significantly reduced in HBOT group at time point $7 \mathrm{~d}$ (Figure 4A). These results suggested that HBOT effectively blocked neural apoptosis pathways. We then measured c-fos and c-jun mRNA level, as expected, HBOT significantly reduced their expression which were most evident at $1 \mathrm{~d}$ post injury, suggesting an early effective window for $\mathrm{HBO}$ treatment (Figure 4B-C).

We speculated that neurotrophic factors (NTFs), known for their function of promoting neuron survival, might play as mediators of the HBOT neural proactive/antiapoptosis effect. By qPCR, we determined the expression of the 4 most abundant NTFs: Brain-derived neurotrophic factor (BDNF), Glial cell line-derived neurotrophic factor (GDNF), nerve growth factor (NGF) and Neurotrophin-3 (NT-3) (Figure 4D-G). As shown in Figure 4F$G$, all the 4 NTFs was elevated after TBI, while HBOT increased their expression further as compared to no-treatment controls, which was most significant at time point $1 \mathrm{~d}$ and continued to day 7. 
A

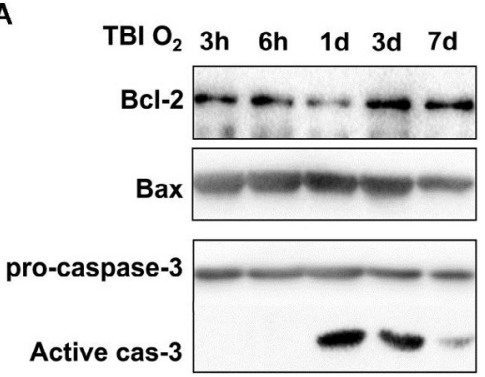

B a

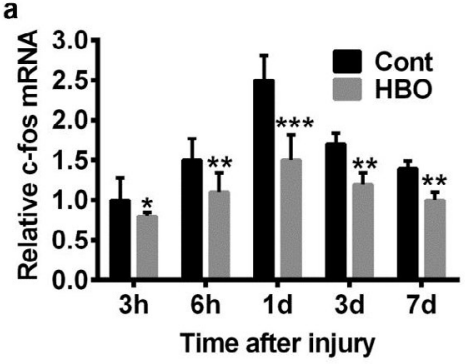

C a

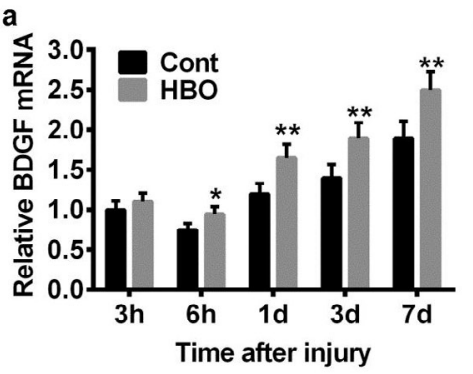

c

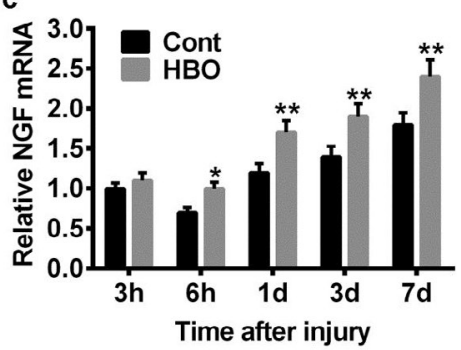

b

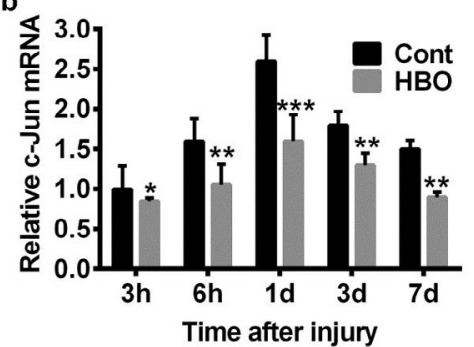

b

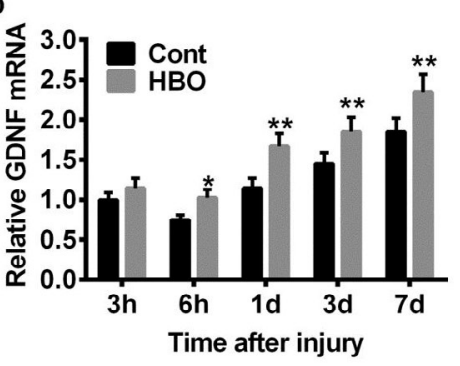

d

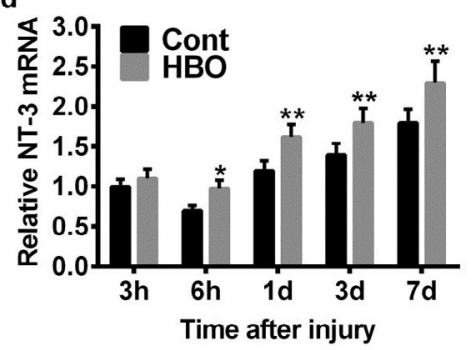

Figure 4 - HBO attenuates the changes of apoptosis genes and promotes the expression of NTFs in vivo. A. Compared with control treatment, HBOT attenuates the reduction of $\mathrm{Bcl}-2$, reduces the increment of Bax and the activation of Caspase-3. B-C. HBOT reduces the mRNA expression increment of c-fos and c-jun throughout the experiment scheme, but most significant at 24 hours after injury. ${ }^{*} p<0.05,{ }^{*} p<0.01, * * * p<0.005$. D-G. The expression of all the checked NTFs (BDNF, GDNF, NGF, NT-3) are elevated after HBOT, which starts at 6 hours after injury and sustains to day $7 .{ }^{*} p<0.05,{ }^{* *} p<0.01$.

Astrocyte is the source of NTFs triggered by TBI-high $\mathrm{O}_{2}$ combination

It is well known that astrocytes support neuronal cells under both physiology and pathology condition by secreting NTFs. We asked whether astrocytes contribute to the increasing expression of NTFs and the neural protection effect of HBOT after TBI. To answer this question, we isolated astrocytes from both $\mathrm{TBI}$ and sham treated rat brains and cultured at both $7 \%$ and $21 \% \mathrm{O}_{2}$ circumstances for 3 days 
before the expression of NTFs were determined. As shown in Figure 5, high $\mathrm{O}_{2}(21 \%)$ promoted the expression of all the checked NTFs in both sham treated and TBI astrocytes, but most significantly in the later. Need to point out was that TBI along is sufficient to activate astrocytes NTFs expression, albeit less significant. These results suggested that astrocytes were activated upon TBI damage to exert its neural supporting/protection function, and hyperoxia further enhanced this activity.

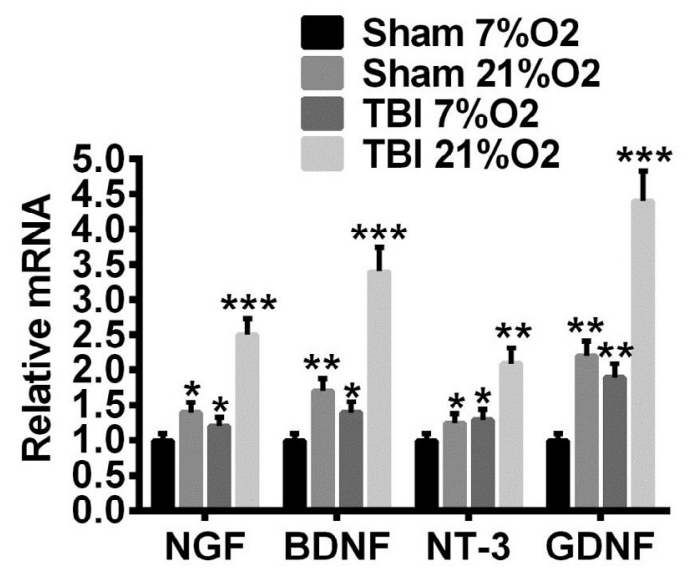

Figure 5 - High oxygen density promotes NTFs expression in cultured astrocytes. After 3 days in vitro culture, the expression of NTFs in astrocytes is elevated in TBI and high $\mathrm{O} 2$ groups compared with sham control or low $\mathrm{O} 2$ groups.

\section{Discussion}

HBO has long been proposed to be an adjunct or enhancement therapy for TBI, but the clinical trials gave controversial results and no conclusions reached so far. In this study, we used Marmarou rat TBI/DTI model revealed that HBOT attenuated neural apoptosis process by reducing the expression of the two immediateearly genes c-fos/c-jun, and re-balancing the ratio of $\mathrm{BCl}-2 / \mathrm{Bax}$, as a consequence, the activation of apoptosis executioner protein
Caspase-3 was subdued. This effect was mediated, at least partially, by the increasing expression of NTFs which we found attributing to the activation of astrocytes.

There are multiple closed head impact models designed to replicate the pathobiology of human concussive and diffuse traumatic injury ${ }^{35}$, among which Marmarou's weight drop model is most wildly used because the device is easy to set up. By modifying the height of dropping weight and constrain secondary injury we achieved moderate TBI model with almost zero skull fracture and mortality, which were reported to be $12.5 \%$ and $44 \%$ respectively in the original study ${ }^{30,33}$. It was reported that during the first 4 hours after injury, reduced cerebral blood flow (CBF) and elevated intracerebral pressure could be observed as the consequence of cerebral autoregulation loss ${ }^{36}$ head injured by weight drop from one meter height using $350 \mathrm{~g}, 400 \mathrm{~g}$ and $450 \mathrm{~g}$ respectively. CBF was monitored using laser-Doppler flowmetry along with monitoring of ICP and arterial blood pressure. If the correlation coefficient between CBF and CPP was $>0.85$ and CPP was within normal range, loss of autoregulation was hypothesized. Loss of autoregulation was seen in all groups of injured rats during first four hours. A statistically significant difference ( $p=0.041$, based on which, it's reasonable to deduce that reduced CBF could further worsen neuropathy caused by primary impact. As comprehensive consequences, weight drop impact induced apoptotic protein $\mathrm{Bcl}-2 / \mathrm{Bax}$ ratio change, activated caspase- 3 and released cytochrome c from mitochondria into cytosol ${ }^{37}$, the former two phenomena were confirmed in this study. It was reported in ischemic wound model, HBOT decreased inflammation and apoptosis by up-regulating $\mathrm{Bcl}-2$ expression but inhibiting Caspase- 3 activity ${ }^{38}$. We found it was also the case for HBO treated rat TBI model. In mouse Neuro2A cells, a nuroblastoma cell line, deprivation of oxygen and glucose 
increased c-fos expression at both mRNA and protein level, and a more interesting finding was that mitochondria recruited excess c-fos protein during the process of cell apoptosis ${ }^{39}$. In mouse retina photoreceptor cells, knockout of c-fos rendered the cells resistance to apoptosis signaling. We found that TBI rapidly stimulated the expression of c-fos and its partner gene $c$-jun. Though the exact function of c-fos in neural death remained unclear, we speculated that it might play a role by forming transcriptional activator complex AP-1 together with c-jun, and regulating the expression of certain genes associated apoptosis. c-jun is a $39 \mathrm{kD}$ protein which could be phosphorylated on multiple serine and threonine sites by JNK. c-jun was reported to play anti-apoptotic roles by inhibit p21 and p53, whether it also functioned as apoptosis inhibitor and how did it balanced with c-fos function during TBI was still unknown. Further studies are needed to determine whether $\mathrm{Bcl}-2$ or Bax was the target of AP-1 transcriptional activation. However, we observed a clear positive correlation among c-fos/c-jun expression, apoptosis effector proteins (Bcl-2/Bax/ Caspase-3) modulation and neural death, suggesting that they were not isolated events, rather there might be inherent relationship unrevealed. HBO treatment also attenuated c-fos/c-jun up-regulation, in a similar trend as that of apoptosis effector proteins, which further indicated such a possibility.

In the health brain, astrocytes are widely spreadedand function to preserve environment for neural circuit function, such as maintaining the homeostasis of ions, transmitters, water, and nutrition. It is well documented that various kinds of damages and diseases can activate astrocytes, which is termed as reactive astrogliosis. In the present study, we found that TBI stimulated the expression of NTFs, which might function as paracrine factors promoting neurons to leave apoptosis pathways and survive damages. We attributed the surge of NTFs to the activation of astrocyte, and confirmed the results in cultured cells, but it was not clear how mechanical forces (weight drop impact) were translated into cell signal that could be recognized by astrocytes and the properly responded. It was reported that astrocytes express mechanotransducing ion channels andstretch-sensitive cation channels on the membrane surface, which contribute to rapid influx of extracellular calcium and sodium upon membrane deformation caused by $\mathrm{TBI}^{40-43}$. The intracellular network formed by glial fibrillary acidic protein (GFAP), the astrocyte specific intermediate filaments, may also participates in transduction of mechanical stretch as it was reported to be up-regulated by trauma ${ }^{44}$. How was the above cell signals translated into the expression up-regulation of NTFs? This issue is rarely discussed and further work is needed. It was interesting that high oxygen density could further elevate the incensement of NTFs expression, which indicated that astrocytes also responded to $\mathrm{O}_{2}$ stimuli in a positive way and this is a strong support for clinical usage of HBOT. The difficulties to developing therapeutic strategies for $\mathrm{TBI}$ is largely lie in the fact that multiple factors are tightly tangled and affect each other during neuropathy progressing. Furthermore, in the case of diffused traumatic brain injury, there is no specific location for targeted treatments.

\section{- Conclusions}

The robust astrocytes in the brain could be activated by traumatic brain injury and following hyperbaric oxygen treatment. Optimized HBOT parameters and treating scheme may better stimulate the protection effect of astrocytes and extend its medical application. 


\section{Reference}

1. Kushner D. Mild traumatic brain injury: Toward understanding manifestations and treatment. Arch Intern Med. 1998;158(15):1617-24. PMID: 9701095.

2. Nguyen R, Fiest KM, McChesney J, Kwon CS, Jette N, Frolkis AD, Atta C, Mah S, Dhaliwal H, Reid A, Pringsheim T, Dykeman J, Gallagher C. The international incidence of traumatic brain injury: a systematic review and metaanalysis. Can J Neurol Sci. 2016;43(6):77485. PMID: 27670907.

3. Langlois JA, Rutland-Brown W, Wald MM. The epidemiology and impact of traumatic brain injury a brief overview. J Head Trauma Rehabil. 2006;21(5):375-8. PMID: 16983222.

4. KimE. Agitation, aggression, and disinhibition syndromes after traumatic brain injury. NeuroRehabilitation. 2002;17(4):297-310. PMID: 12547978.

5. Smith DH, Meaney DF, Shull HW. Diffuse axonal injury in head trauma. J Head Trauma Rehabil. 2003;18(4):307-16. PMID: 16222127.

6. Granacher Jr RP. Traumatic brain injury. Methods for clinical \& forensic neuropsychiatric assessment. 2ed. CRC Press; 2007:32.

7. Kraus MF, Susmaras T, Caughlin BP, Walker CJ, Sweeney JA, Little DM. White matter integrity and cognition in chronic traumatic brain injury: a diffusion tensor imaging study. Brain. 2007;130(10):2508-19. PMID: 17872928.

8. Kumar R, Husain M, Gupta RK, Hasan KM, Haris M, Agarwal AK, Pandey CM, Narayana PA. Serial changes in the white matter diffusion tensor imaging metrics in moderate traumatic brain injury and correlation with neuro-cognitive function. J Neurotrauma. 2009;26(4):481-95. PMID: 19196176.

9. Janowitz T, Menon DK. Exploring new routes for neuroprotective drug development in traumatic brain injury. Sci Transl Med. 2010;2(27):27rv1. PMID: 20393189.

10.Gruenbaum SE, Zlotnik A, Gruenbaum BF, Hersey D, Bilotta F. Pharmacologic neuroprotection for functional outcomes after traumatic brain injury: a systematic review of the clinical literature. CNS Drugs. 2016;30(9):791-806. PMID: 27339615.
11. Holleran L, Kim JH, Gangolli $M$, Stein T, Alvarez V, McKee A, Brody DL. Axonal disruption in white matter underlying cortical sulcus tau pathology in chronic traumatic encephalopathy. Acta Neuropathol. 2017;133(3):367-80. PMID: 28214960.

12.Finkelstein EA, Corso PS, Miller TR. The incidence and economic burden of injuries in the United States. New York: Oxford University Press; 2006.

13.Coronado VG, McGuire LC, Sarmiento K, Bell J, Lionbarger MR, Jones CD, Geller Al, Khoury $\mathrm{N}, \mathrm{Xu} \mathrm{L}$. Trends in traumatic brain injury in the U.S. and the public health response: 1995-2009. J Safety Res. 2012;43(4):299307. PMID: 23127680.

14. Hay J, Johnson VE, Smith DH, Stewart W. Chronic traumatic encephalopathy: the neuropathological legacy of traumatic brain injury. Annu Rev Pathol Mech Dis. 2016;11(1):21-45. PMID: 26772317.

15.Edwards ML. Hyperbaric 02 therapy. Part 2: Application in disease. J Vet Emerg Crit Care. 2010;20(3):289-297. PMID: 20636981.

16. Huang L, Obenaus A. Hyperbaric oxygen therapy for traumatic brain injury. Med Gas Res. 2011;1(21). PMID: 22146562.

17.Zhilyaev SY, Moskvin AN, Platonova TF, Gutsaeva DR, Churilina IV, Demchenko IT. Hyperoxic vasoconstriction in the brain is mediated by inactivation of nitric oxide by superoxide anions. Neurosci Behav Physiol. 2003;33(8):783-7. PMID: 14635993.

18.Skyhar MJ, Hargens AR, Strauss MB, Gershuni $\mathrm{DH}$, Hart GB, Akeson WH. Hyperbaric oxygen reduces edema and necrosis of skeletal muscle in compartment syndromes associated with hemorrhagic hypotension. J Bone Jt Surg Am. 1986;68(8):1218-24 . PMID: 3021776.

19.Ostrowski RP, Colohan AR, Zhang JH. Mechanisms of Hyperbaric oxygeninduced neuroprotection in a rat model of subarachnoid hemorrhage. J Cereb Blood Flow Metab. 2005;25(5):554-71. PMID: 15703702.

20.Gungor A, Poyrazoglu E, Cincik H, Sali M, Candan $\mathrm{H}$. The effectiveness of hyperbaric oxygen treatment in tracheal reconstruction with auricular cartilage grafts (experimental study). Am J Otolaryngol. 2003;24(6):390-4 . PMID: 14608571.

21.Zhang T, Gong W, Li Z, Yang S, Zhang K, Yin 
D, Xu P, Jia T. Efficacy of hyperbaric oxygen on survival of random pattern skin flap in diabetic rats. Undersea Hyperb Med. 2007;34(5):335-9. PMID: 18019084.

22.Jiang Z, Li X, Li Y, Peng L, Wang G, Wang $Y$. Neuroprotective effects of hyperbaric oxygen treatment on traumatic brain injury in the rat. J Neurotrauma. 2010;27(9):1733. PMID: 20568957.

23.Niklas A, Brock D, Schober R, Schulz A, Schneider D. Continuous measurements of cerebral tissue oxygen pressure during hyperbaric oxygenation - HBO effects on brain edema and necrosis after severe brain trauma in rabbits. J Neurol Sci. 2004;219(12):77-82. PMID: 15050441.

24.Calvert JW, Zhou C, Nanda A, Zhang JH. Effect of hyperbaric oxygen on apoptosis in neonatal hypoxia-ischemia rat model. J Appl Physiol. 2003;95(5):2072-80. PMID: 14555671.

25.Abrous DN, Rodriguez J, le Moal M, Moser PC, Barnéoud P. Effects of mild traumatic brain injury on immunoreactivity for the inducible transcription factors c-Fos, c-Jun, JunB, and Krox-24 in cerebral regions associated with conditioned fear responding. Brain Res. 1999;826(2):181-92. PMID: 10224295.

26.Dave JR, Bauman RA, Long JB. Hypoxia potentiates traumatic brain injury-induced expression of c-fos in rats. Neuroreport. 1997;8(2):395-8. PMID: 9080414.

27.Wang $Y$, Hameed MQ, Rakhade SN, Iglesias AH, Muller PA, Mou DL, Rotenberg A. Hippocampal immediate early gene transcription in the rat fluid percussion traumatic brain injury model. Neuroreport. 2014:1-6. PMID: 24978397.

28.Villasana LE, Westbrook GL, Schnell E. Neurologic impairment following closed head injury predicts post-traumatic neurogenesis. Exp Neurol. 2014;261:15662. PMID: 24861442.

29.Cernak I, Vink R, Zapple DN, Cruz MI, Ahmed F, Chang T, Fricke ST, Faden Al. The pathobiology of moderate diffuse traumatic brain injury as identified using a new experimental model of injury in rats. Neurobiol Dis. 2004;17(1):29-43. PMID: 15350963.

30.Marmarou A, Foda MA, van den Brink W, Campbell J, Kita H, Demetriadou K. A new model of diffuse brain injury in rats. Part
I: Pathophysiology and biomechanics. J Neurosurg. 1994;80(2):291-300. PMID: 8283269.

31. Flierl MA, Stahel PF, Beauchamp KM, Morgan SJ, Smith WR, Shohami E. Mouse closed head injury model induced by a weightdrop device. Nat Protoc. 2009;4(9):1328-37. PMID: 19713954.

32.Souza DG, Bellaver B, Souza DO, QuincozesSantos A. Characterization of adult rat astrocyte cultures. PLoS One. 2013;8(3):110. PMID: 23555943.

33. Foda MA, Marmarou A. A new model of diffuse brain injury in rats. Part II: morphological characterization. J Neurosurg. 1994;80(2):301-13. PMID: 8283270.

34.Clark RSB, Kochanek PM, Watkins SC, Chen M, Dixon CE, Seidberg NA, Melick J, Loeffert JE, Nathaniel PD, Jin KL, Graham SH. Caspase-3 mediated neuronal death after traumatic brain injury in rats. J Neurochem. 2000;74(2):740-53. PMID: 10646526.

35. Cernak I. Animal models of head trauma. NeuroRx. 2005;2(3):410-22. PMID: 16389305.

36.Prat R, Markiv V, Dujovny M, Misra M. Failure of cerebral autoregulation in an experimental diffuse brain injury model. Acta Neurochir Suppl. 1998;71:123-6. PMID: 9779163.

37.Cernak I, Chapman SM, Hamlin GP, Vink R. Temporal characterisation of pro- and antiapoptotic mechanisms following diffuse traumatic brain injury in rats. J Clin Neurosci. 2002;9(5):565-72. PMID: 12383417.

38.Zhang Q, Chang Q, Cox RA, Gong X, Gould LJ. Hyperbaric oxygen attenuates apoptosis and decreases inflammation in an ischemic wound model. J Invest Dermatol. 2008;128(8):2102-12. PMID: 18337831.

39. Kambe Y, Miyata A. Mitochondrial c-Fos may increase the vulnerability of neuro2a cells to cellular stressors. J Mol Neurosci. 2016;59(1):106-12. PMID: 26768136.

40. Bowman CL, Ding JP, Sachs F, Sokabe M. Mechanotransducing ion channels in astrocytes. Brain Res. 1992;584(1-2):27286. PMID: 1381266.

41.Islas L, Pasantes-Morales H, Sanchez JA. Characterization of stretch-activated ion channels in cultured astrocytes. Glia. 1993;8(2):87-96. PMID: 8406677.

42.Rzigalinski BA, Weber JT, Willoughby KA, 
Ellis EF. Intracellular free calcium dynamics in stretch-injured astrocytes. J Neurochem. 1998;70(6):2377-85. PMID: 9603202.

43.Floyd CL, Gorin FA, Lyeth BG. Mechanical strain injury increases intracellular sodium and reverses $\mathrm{Na}+/ \mathrm{Ca} 2+$ exchange in cortical astrocytes. Glia. 2005;51(1):35-46. PMID:
15779085.

44. Liu Z, Li Y, Cui Y, Roberts C, Lu M, Wilhelmsson $U$, Pekny M, Chopp M. Beneficial effects of gfap/vimentin reactive astrocytes for axonal remodeling and motor behavioral recovery in mice after stroke. Glia. 2014;62(12):202233. PMID: 25043249.

\section{Correspondence:}

Ke Ma

Department of Emergency and Intensive Care Unit

Shanghai Sixth People's Hospital East

Shanghai, 201306 China

makewy08@163.com

Received: Dec 27, 2017

Review: Feb 24, 2018

Accepted: Mar 23, 2018
Conflict of interest: none

Financial source: Courtyard Scientific Research Fund of Shanghai Sixth People's Hospital East (grant numbers 2016025). 